\title{
Testicular Plasmacytoma in a Patient with Multiple Myeloma
}

\author{
DS Kahraman ${ }^{1}, \mathrm{~K}$ Yorukoglu ${ }^{2}, \mathrm{M} \mathrm{Kilic}^{1}, \mathrm{~N}$ Erbil $^{1}, \mathrm{H} \mathrm{Ucok}^{3}$ \\ Departments of ${ }^{1}$ Pathology and ${ }^{3}$ Urology, İzmir Health Sciences University, Tepecik Training and Research Hospital, Izmir, Turkey; \\ ${ }^{2}$ Department of Pathology, Dokuz Eylül University, Medical Faculty Hospital, Izmir, Turkey.
}

\section{Corresponding Author:}

Dr. Dudu Solakoglu Kahraman

Email: dudusolakoglu@hotmail.com

This is an Open Access article distributed under the terms of the Creative Commons Attribution License (creativecommons.org/ licenses/by/3.0).

Received Accepted

Published

March 1, 2019

August 17, 2019

August 25, 2019

\begin{abstract}
Background: Testicular plasmacytoma's are rarely seen neoplasia and they can occur as solitary plasmacytomas, a late relapse of multiple myeloma (MM), or concurrently with an active myeloma. Case Report: We report the case of a 73-year-old man who presented with testicular mass and diagnosed with plasmacytoma. The patient's history revealed that he had been treated for MM. He presented with a gradually enlarging scrotal mass. Following orchidectomy, pathologic examination of the specimen was reported as plasmacytoma. Conclusion: The clinicopathological significance of testicular localization of $\mathrm{MM}$ is discussed in the light of the literature.
\end{abstract}

Keywords: Multiple Myeloma, Orchiectomy, Plasma Cell Neoplasms, Plasmacytoma, Testis.

\section{Introduction}

Extramedullary plasmacytoma (EMP) is often accompanied by infiltration of bone marrow with plasmacytes, while it is rarely seen during the natural course of multiple myeloma. EMP is often localized on the head. Testicular localization is rare $[1,2]$. Non-germinative cell testicular tumors $(5 \%)$, and plasma cell neoplasia's $(2 \%)$ account only for a small percentage of testicular plasmacytoma [3]. The presence of testicular plasmacytoma shows widespread disease and has a poor prognosis $[1,2]$. Testicular plasmacytomas may occur at the onset of relapsing multiple myeloma or as a common disease when the disease first develops $[2,4]$.

\section{Case Report}

A 73-year-old male had noticed swelling and pain in his right scrotum existing for one and half months. Physical examination confirmed the presence of right testicular mass. The right testicle was found to be enlarged at scrotal colour Doppler ultrasonography. There was a hypoechoic lesion with increased vascularity suggesting a malignant tumor. Radical inguinal orchiectomy was performed. The macroscopic examination of the specimen showed a relatively confined, homogenous cream-colored tumoral lesion of $4 \times 4 \times 3.5 \mathrm{~cm}$ in size. Tunica albuginea resisted the spread of the tumor, and tunica vaginalis and epididymis were intact.

Microscopically, there was a diffuse interstitial atypical plasma cell infiltration sparing the seminiferous tubules with hypospermatogenesis [Fig 1,2]. Immunohistochemistry showed that the tumor cells were positive for CD138 [Fig.3], lambda light chains, monoclonal cytoplasmic production of $\mathrm{IgG}$, and epithelial membrane antigen (EMA) and negative for placental alkaline phosphatase (PLAP) and leukocyte common antigen (LCA). The patient had received the diagnosis of multiple myeloma at an external center 9 years ago. A final diagnosis of plasmacytoma was made. After the diagnosis of testicular plasmacytoma was made, the patient received 20 cycles of radiotherapy. The patient is under regular follow-up for one year, has no active complaints at the moment. 


\section{Discussion}

Testicular plasmacytomas have been identified in multiple settings, mostly involving patients with concurrent MM. As in our case, testicular EMP has also been reported at a recurrence site of multiple myeloma during remission of MM [5]. In rare cases, the plasmacytoma of the testes may occur without previously detected hematological malignancy $[2,6,7]$. Unhappily, most of these patients will develop multiple myeloma after only a few longterm progression free survival after orchiectomy [3]. Testicular interstitial infiltration with atypical cells warrants differential diagnosis of seminoma, spermatocytic tumor, malignant lymphoma, malignant melanoma, and plasmacytoma [8].

As in our case, we could establish the diagnosis based on clinical and histomorphological findings and immunohistochemical staining results. Even though the most accepted treatment for extramedullary plasmacytomas is radiotherapy, orchiectomy has been the preferred treatment in almost all case reports of testicular tumors probably due to low level of suspicion from EMPs [9-11]. This particular natural behavior presented by the testicular plasmacytoma may lead to a diagnostic challenge, because the clinical differential diagnosis with other benign diseases cannot be made precisely and other malignancies cannot be safely excluded without histopathological examination. Orchiectomy is the main treatment of choice over radiotherapy in almost all cases due to lack of high level of suspicion from testicular EMPs. Despite of advances in treatment, patients have a poor prognosis $[3,12]$.

\section{Conclusion}

We present a rare case of testicular EMP in a patient with multiple myeloma. The differential diagnosis of testicular plasmacytoma includes wide range of tumors with very different treatment strategies. Testicular involvement of a multiple myeloma patient may not necessitate radical

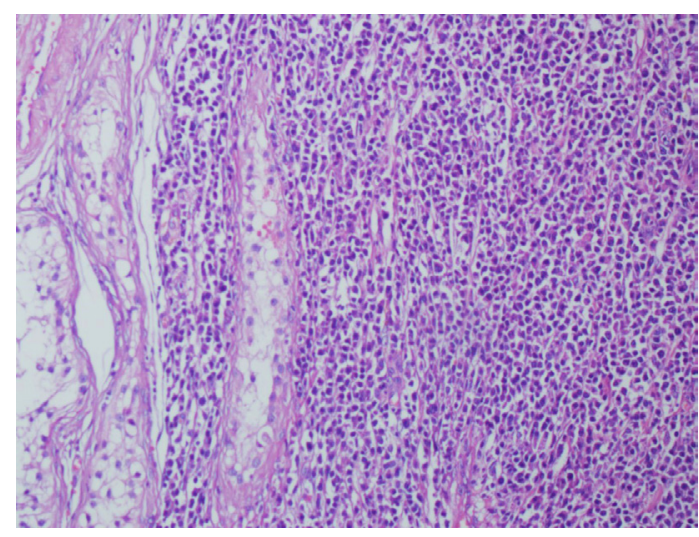

Fig.1: Histopathology of testicular plasmacytoma $(H \& E \times 100)$.

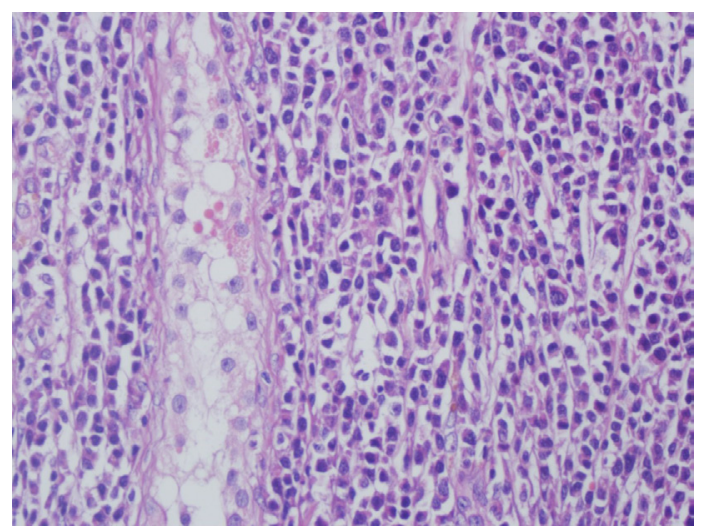

Fig.2: Microscopically tumor shows diffuse infiltration atypical plasma cells with eccentrically located nuclei and the residual seminiferous tubules separated by heavy infiltrates of plasma cells $(H \& E \times 200)$.

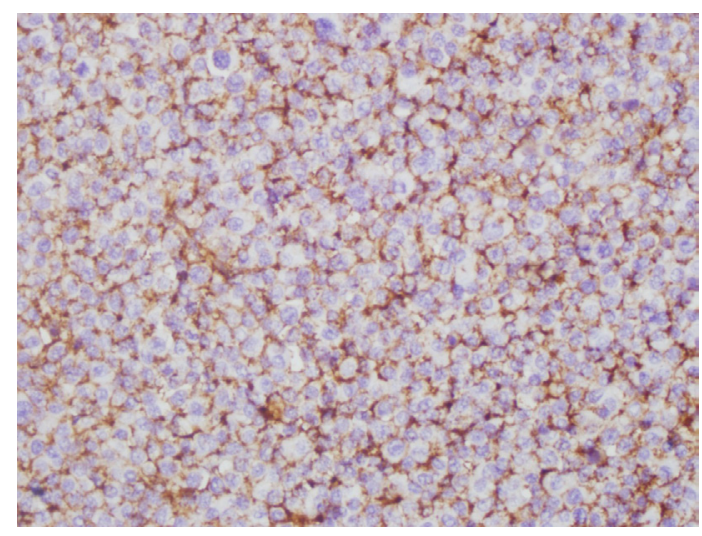

Fig.3: Immunohistochemical stains of surgical specimen obtained from right orchiectomy show positive reaction for CD138 in plasma cells $(D A B \times 400)$. 
orchiectomy. Therefore, any testicular mass requires a full diagnostic workup before radical treatment, especially in patients without of the age range of germ cell tumors. Primary plasmacytoma prognosis is poor and progression to multiple myeloma is likely. Any interstitial infiltration with malignant cells indicates a wide range of differential diagnosis with both morphological and immunohistochemical analysis.

Acknowledgement: This text has been edited by Gurkan Kazanci, a professional translator from Logos Publishing. Contributors: All authors contributed to the development of the review, the design of the figures and in writing the manuscript. DSK, KY and MK participated in the design of the study. NE and HU conceived the study, and participated in its design and coordination and helped to draft the manuscript. DSK will act as a study guarantor. All authors read and approved the final manuscript.

Funding: None; Competing interests: None stated.

\section{References}

1. Bataille R, Harousseau JL. Multiple myeloma. N Engl J Med. 1997;336:1657-1664.

2. Hathaway AR. Incidental discovery of a testicular plasmacytoma at initial presentation of multiple myeloma. Case Rep Hematol. 2013;2013:752921.

3. Filho MG. Primary testicular plasmocytoma: A five year follow-up. Urol Annal. 2013;5:39-41.
4. Rajkumar SV. Multiple myeloma: 2011 update on diagnosis, risk-stratification, and management. American Journal of Hematology. 2011;86:57-65.

5. Rosenberg S, Shapur N, Gofrit O, Or R. Plasmacytoma of the testis in a patient with previous multiple myeloma: Is the testis a sanctuary site? J Clin Oncol. 2010;28:e456-e458.

6. Ferry JA, Young RH, Scully RE. Testicular and epididymal plasmacytoma: A report of 7 cases, including three that were the initial manifestation of plasma cell myeloma. Am J Surg Pathol. 1997;21:590-598.

7. Melicow MM, Cahill GF. Plasmacytoma (multiple myeloma) of testis: A report of four cases and review of the literature. J Urol. 1954;71:103-113.

8. Tanagho Y, Stovsky M, Maclennan GT. Testicular plasmacytoma. J Urol. 2010;184:1161-1162.

9. Soutar R, Lucraft H, Jackson G, Reece A, Bird J, Low $\mathrm{E}$, et al. Guidelines on the diagnosis and management of solitary plasmacytoma of bone and solitary extramedullary plasmacytoma. Clin Oncol (R Coll Radiol). 2004;16(6):405-413.

10. Dimopoulos MA, Hamilos G. Solitary bone plasmacytoma and extramedullary plasmacytoma. Curr Treat Options Oncol. 2002;3(3):255-259.

11. Alexiou C, Kau RJ, Dietzfelbinger H, Kremer M, Spiess JC. Extramedullary plasmacytoma: Tumor occurrence and therapeutic concepts. Cancer. 1999;85:2305-2314.

12. Shimokihara K, Kawahara T, Chiba S, Takamoto D, Yao M, Uemura H. Extramedullary plasmacytoma of the testis: A case report. Urology Case Reports. 2018;16:101103. 